\title{
3D Technologies for Measurement of Painting Surface Deformations: A Case Study
}

\author{
Francesca Uccheddu \\ University of Florence \\ Via S. Marta 3 Firenze, Italy \\ francesca.uccheddu@unifi.it
}

\author{
Anna Pelagotti \\ Istituto Nazionale di Ottica, CNR, \\ Largo Fermi 6, 50125 - Firenze, Italy \\ anna.pelagotti@ino.it \\ Emanuela Massa \\ Art-Test \\ via del Ronco 12 Firenze, Italy \\ massa@art-test.com
}

\author{
Vito Cappellini \\ University of Florence \\ Via S. Marta 3 Firenze, Italy \\ vito.cappellini@unifi.it
}

\begin{abstract}
Ancient painted wooden panels are an important part of our cultural heritage. Wood as a support has always been very popular and painted panels are currently present, in a large number, in many churches and museums. However, depending on conservation conditions, their shape may modify in time and if not properly controlled may lead to some sever damage to the artworks. The paper describes the results of a study on the measurement of paintings surface deformation carried out using two different commercial devices both making use of structured light. The main goal was to highlight and measure the reliability of the such 3D measuring techniques to evaluate deviations from planarity due to the curving and warping of the wood in order to document spatial deformation suffered by the painting and monitoring its conservation status.
\end{abstract}

3D survey. Artwork analysis. Restoration. 3D scanner. Kinect sensor.

\section{INTRODUCTION}

Paintings on wooden panels have been very popular especially until the XV century and are currently present, in a large number, in many churches and museums. However, such environments are only rarely equipped with climate control systems and, therefore, often such artefacts suffer there from highly variable climatic conditions. Being wood a "living" material, it responds to different temperatures and humidity values and changes its shape. Such variations, which sometimes happen over a short period of time, induce stresses and strains that can, in the long term, irreversibly damage the painting. In the past, it was believed that such movements had to be totally avoided and therefore a fixed frame was attached to the back of the panel. However, time has proven this theory wrong, and sometimes, as in the case presented here, the wooden frame applied during a previous restoration intervention has to be removed. In order to control the changes induced by this operation and to quantify the strain and stress applied to the wooden frame panel over time, shape measurements are proposed to analyse how the panel modifies itself. Such analyses could allow researchers and restorers to evaluate and prevent possible panel damages and help them in choosing the most suitable technique to control such movements. Such possibility is therefore envisaged to raise large interest, provided that it is possible to achieve it at reasonable operational costs.

The use of three-dimensional scanning technology (Remondino et al. 2011, Guidi et al. 2004, Robson et al. 2004), so far applied to Cultural Heritage mainly for modeling of sculptural artworks, can provide a detailed map and a quantitative measurement of the painting deterioration, however the case studies presented in literature are mainly reporting on highly costly equipment results, and no comparison before and after an induced deformation are described.

The main purpose of the study was to evaluate the relative accuracy, and the reliability, of two 3D devices, (and of the commercial software provided to shape the model), belonging to very different price categories. The two different technologies tested were both based on structured light projection, one in the visible range and the other in the infrared range 


\section{EXPERIMENTAL SETUP}

The case study was a very large $(210 \mathrm{~cm} \times 190 \mathrm{~cm})$ $\mathrm{XV}$ century panel.

In a previous restoration treatment, three horizontal wooden battens were inserted to the back of the panel with the intent of flattening the artwork (as in Figure 1). Nowadays restores deemed important to remove such constraints in order to give back to the wooden panel the original freedom to move spontaneously, although in a controlled environmental conditions.
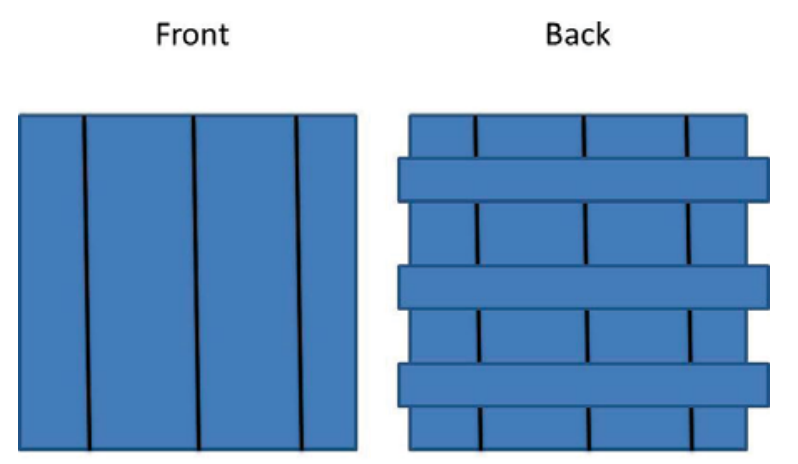

$$
\begin{array}{cc}
\begin{array}{c}
\text { Wooden } \\
\text { boards }
\end{array} & \begin{array}{c}
\text { Wooden } \\
\text { battens }
\end{array}
\end{array}
$$

Figure 1: The front of the artwork is painted on some main wooden boards. On the back the three battens

The wooden support was expected to move after the battens removal, however none could predict of how much. Such a parameter is instead crucial to assess the deformation and monitoring its evolution in order to allow timely intervention before irreversible damage can take place.

The work here presented, aimed at comparing the performance of two difference devices, belonging to very difference price ranges. Moreover, a first survey was performed before removing the wooden battens, and a second one afterwards, in order to evaluate the induced deformation.

The first device tested was a structured light based scanner, (Figure 2) ScanProbe LT by Scan Systems, while the second was a Kinect infrared sensor system (Figure 3).

The first scanner, working according to a method described in literature (Salvi et al. 2010), makes use of a 3D triangulation system based on projecting onto the object under study eight patterns, that is, images composed by alternating black and white vertical stripes, at increasing frequency. The stripes projected on the surface warp depending on the surface surveyed. A camera records the images which are then processed.
Projector, camera and pattern's points represent the vertices of a triangle in the space and, therefore, the spatial position of the points on the profile is derived according to the known triangulation method. The price range of such sort of scanners is around the 20000 euros.

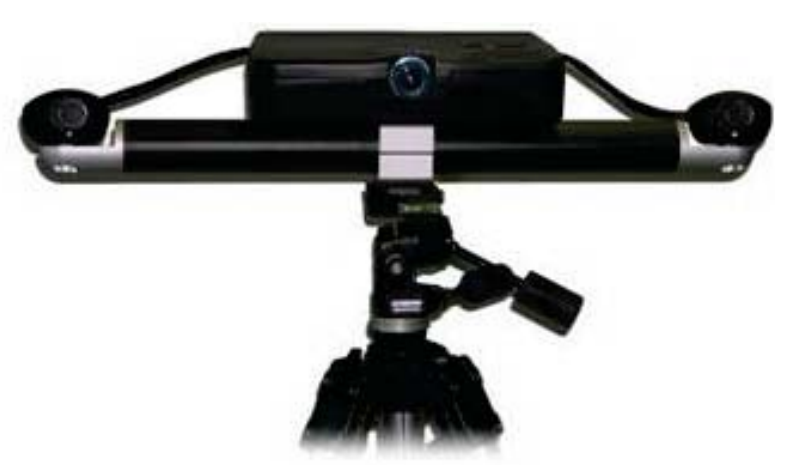

Figure 2: The visible structured light based scanner ScanProbe $L T$ by ScanSystems

The second scanner (Kinect sensor, Figure 3) consists of an infrared laser emitter, an infrared camera and an RGB camera (Khoshelham et al. 2012). The measurement of depth is a triangulation process in which the laser source emits a single beam which is split into multiple beams by a diffraction grating to create a constant pattern of speckles projected onto the scene. This pattern is captured by the infrared camera and is correlated to a reference pattern. When a speckle pattern is projected onto an object of which the distance from the sensor is smaller or larger than that of a known reference plane, the position of the speckle in the infrared image captured will be shifted in the direction of the baseline between the laser projector and the perspective center of the infrared camera. These shifts are measured for all speckles by a simple image correlation procedure, which yields a disparity image. For each pixel the distance to the sensor can then be retrieved from the corresponding disparity. Such system costs currently around 250 euros.

Since a single range map taken at high resolution covers a limited surface, the number of images to be merged together in order to generate a complete three-dimensional model of the artwork has to be increased as the resolution grows.

Another element to be taken into account is represented by measurement uncertainty, which depends on the squared distance between the camera and the object: a high precision is achieved by suitably limiting this distance, and thus the framed area.

For the first acquisition campaign and the ScanProbe device, the scanner was approximately $90 \mathrm{~cm}$ far from the painting and 105 range maps 
were achieved to cover the entire area in 6 hours as shown in Figure 4. Much longer was the processing time, making this procedure hard to be proposed as routine monitoring.

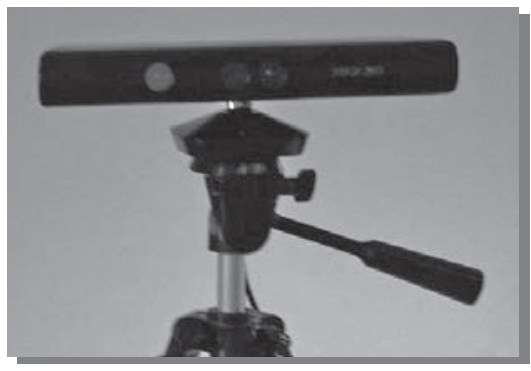

Figure 3: The infrared Kinect sensor

The main difficulty was then related to the way alignment algorithms work to find a relative orientation of two adjacent range maps. In order to refer all the acquisitions to the same reference system, adjacent range maps are aligned by means of specific software procedures that minimize the mean square deviation or find a best match between overlapping points.

Such procedures, indicated in the literature as Iterative Closest Point (ICP) algorithms (Segal et al. 2009), use a cost function, for example consisting of the mean square deviation between groups of homologous points, that have to be minimized starting from a pre-alignment manually imposed by an operator. The function value decreases steeply to a well recognisable absolute minimum in the case of surfaces with evident 3D features such as bumps or extrusions. It becomes less peaky, with many local minima, when the surfaces to be aligned have no evident $3 \mathrm{D}$ details, as on the flat surface of the front painting side. In the latter case the manual pre-alignment is a crucial step that influence the failure/success of the final convergence, the minimisation becomes also much slower. Since in this case the aimed precision was not less than one centimetre, a decimated version of the 3D model was considered to perform such registration. When the model is shown without texture, the shape of the underlying wooden structure is highlighted (as in Figure 5).

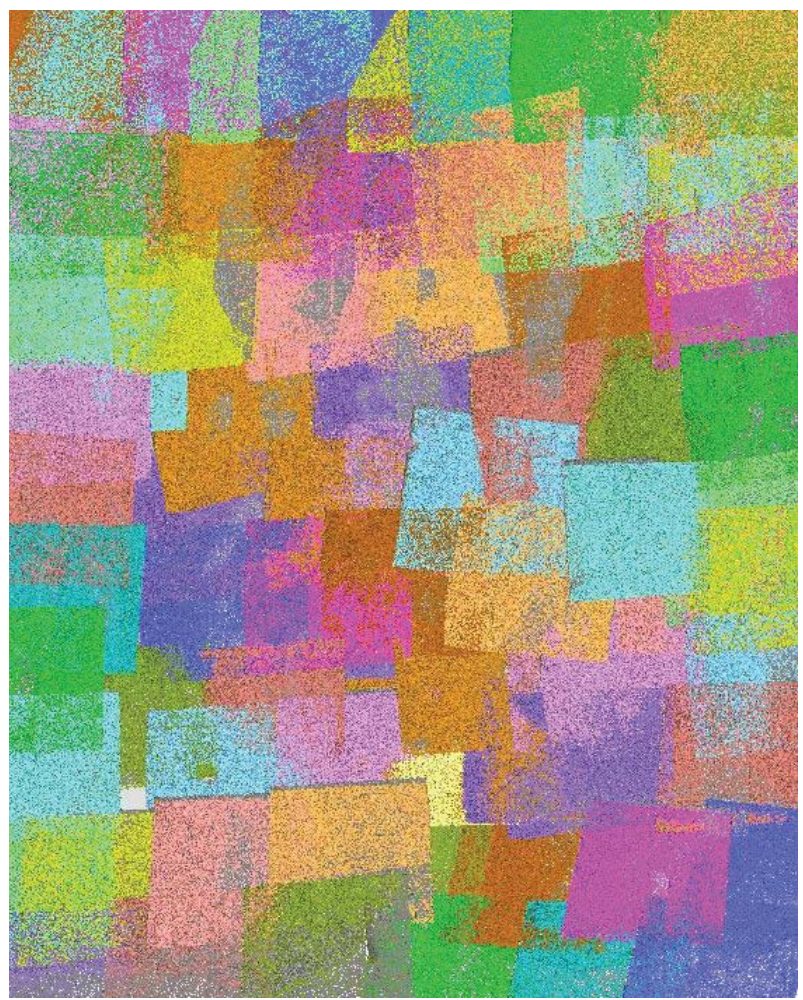

Figure 4: The wooden painting $(210 \mathrm{~cm} \times 190 \mathrm{~cm})$, surveyed with 105 scans. The final 3D model contains approximately 50 million faces

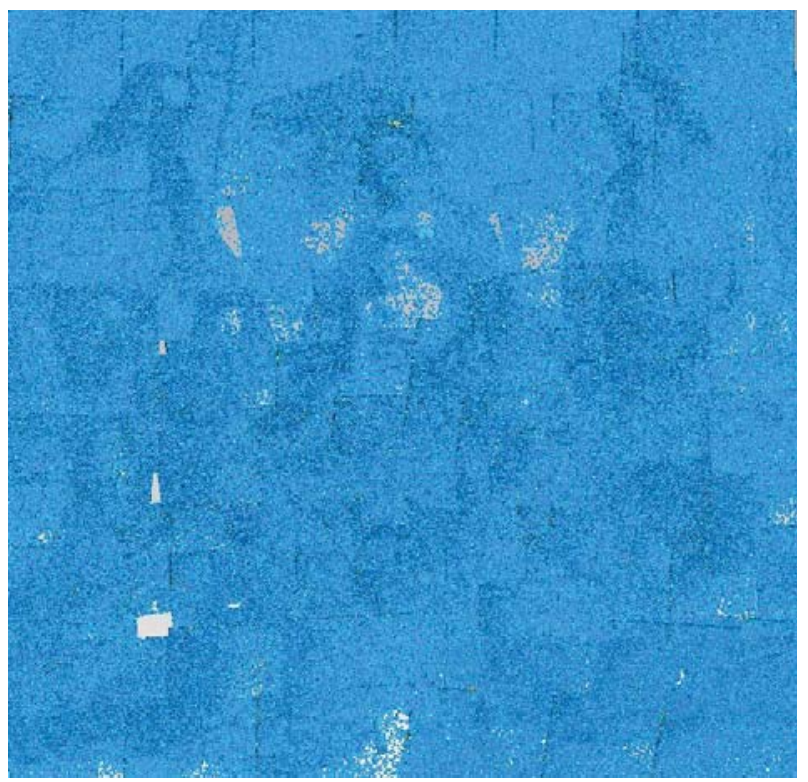

Figure 5: The complete decimated 3D model containing 5millions triangles 
After the battens were removed, we then scanned the artwork both with the Scanprobe and with the Kinect infrared sensor system. In this second acquisition campaign the scanner was positioned 3 meters far from the painting. This allowed to obtain in a couple of seconds the model reported in Figure 6.

The Kinect sensor has the advantage of having a very big field of view thus allowing a very fast acquisition of the entire painting. In our experiment since the area to be covered was more than $2 \mathrm{~m} x$ $2 \mathrm{~m}$, a distance of about 3 meters was needed to cover it fully (see Figure 6).

This sort of test would be the cheapest, easiest and fastest way, for a trained nonprofessional, to achieve a 3D model of such a panel painting.

The accuracy cannot be the same as with the previous acquisition, not only because of the less accurate system but also because for every device, the accuracy of the measured data decrease with the squared distance (see Figure 7).

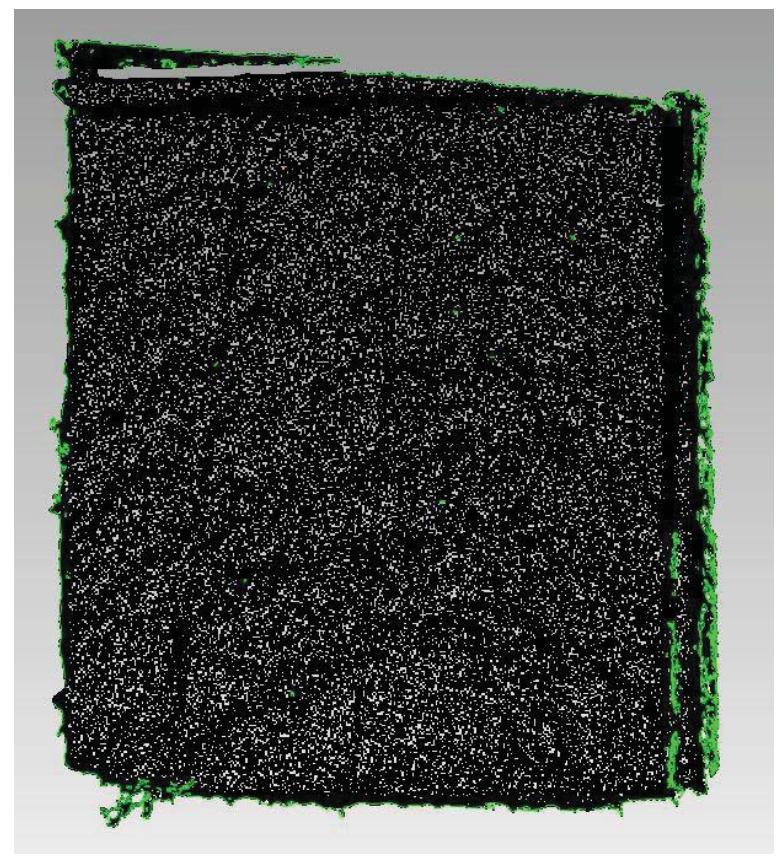

Figure 6: The complete Kinect sensor acquisition of the painting

\section{RESULTS}

In order to perform a quantitative analysis, once the models were generated they were used to evaluate the painting deformations with respect to a planar reference. The reference was created by identifying the best-fitting plane of the cloud of points associated to the 3D model. The painting deformations, represented by the distances of the model points with respect to the reference plane, were thus displayed using color coding.
The hardest challenge was put on the Kinect system, intentionally so, in order to test its limits.

The acquisition performed with the ScanProbe device before the battens removal, shows, as expected, a rather flat surface.

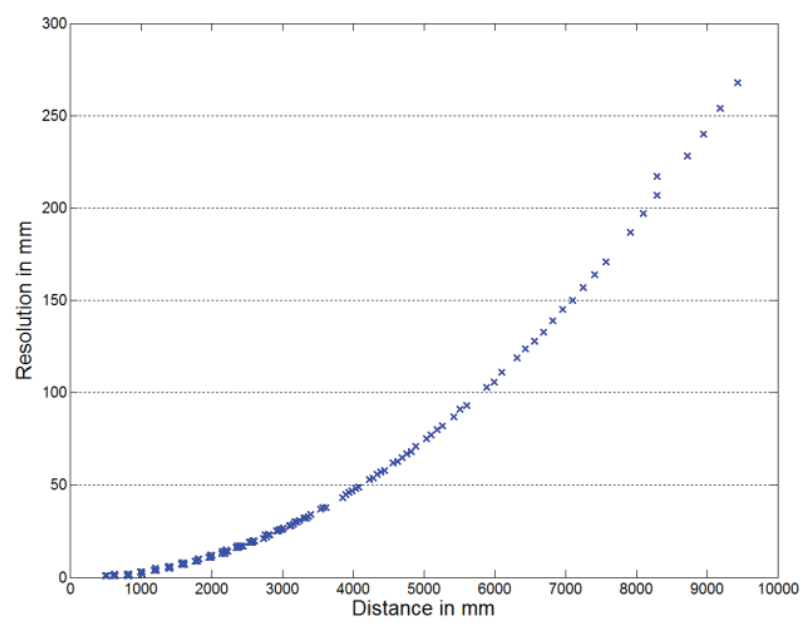

Figure 7: The depth resolution is a function of distance and is getting coarser as the distance increases

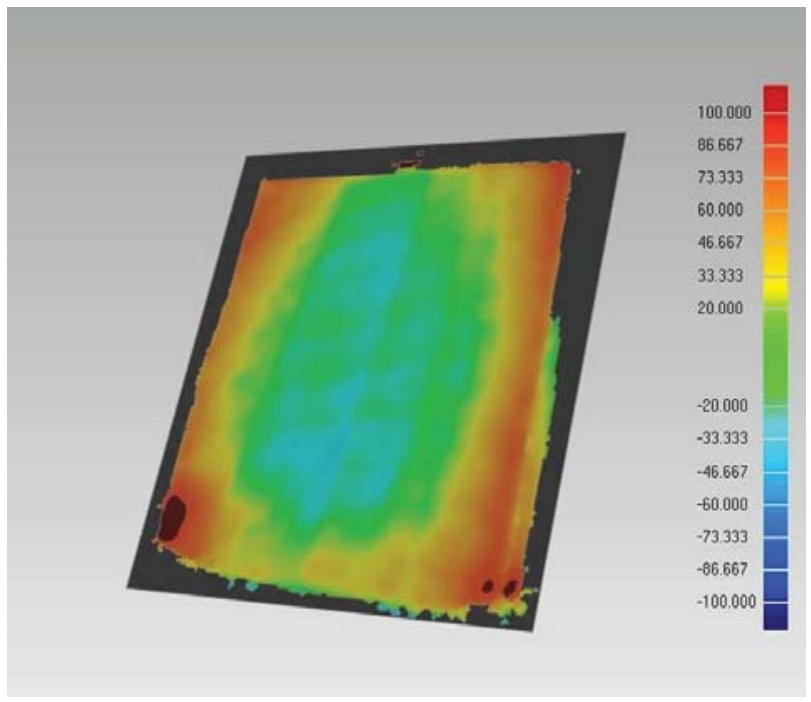

Figure 8: The Kinect sensor acquisition compared to an ideal plane shows that depth estimate varies in the image as a circular ripple

In Figure 9 is depicted the deviations from a local best-fit plane in the four corners of the painting (as we supposed, the corners are the more stressed areas), as acquired with the ScanProbe scanner. Only small variations $(+/-3 \mathrm{~mm}$ almost everywhere) from local planarity is observed and the vertical wooden board are slightly visible.

Figure 11 shows the other areas where the largest deformation is to be expected. 

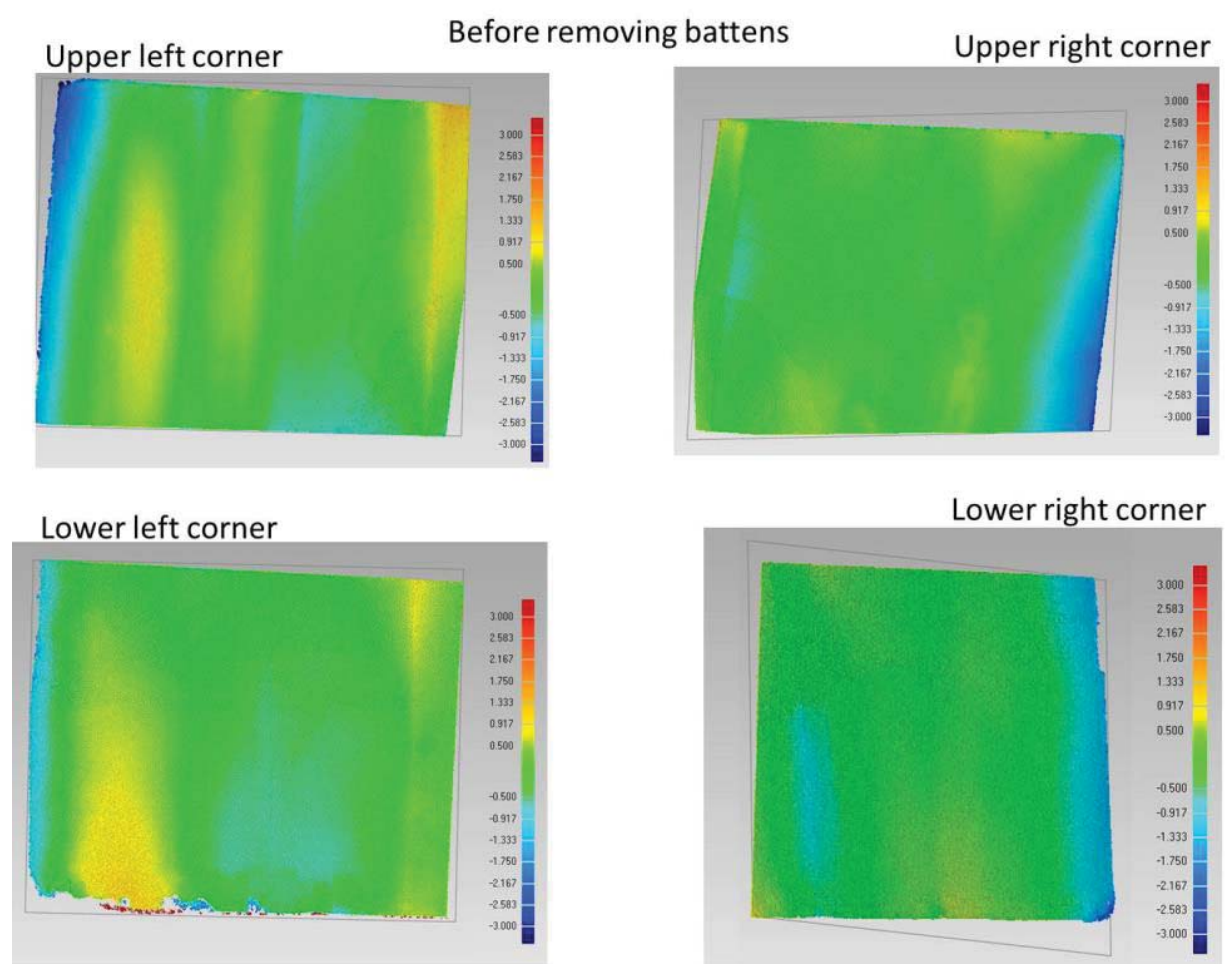

Lower right corner

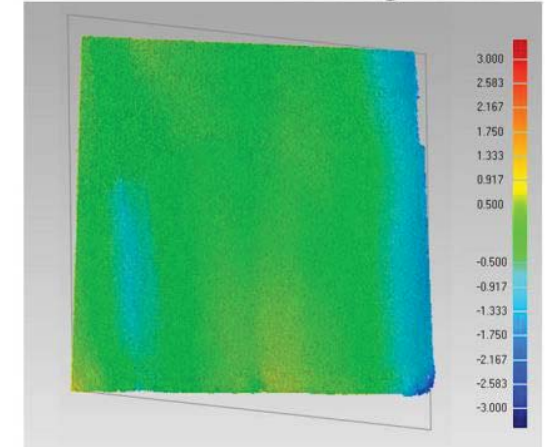

Figure 9: Image of deviations $(\mathrm{mm})$ from the best-fit plane before removing the wooden battens
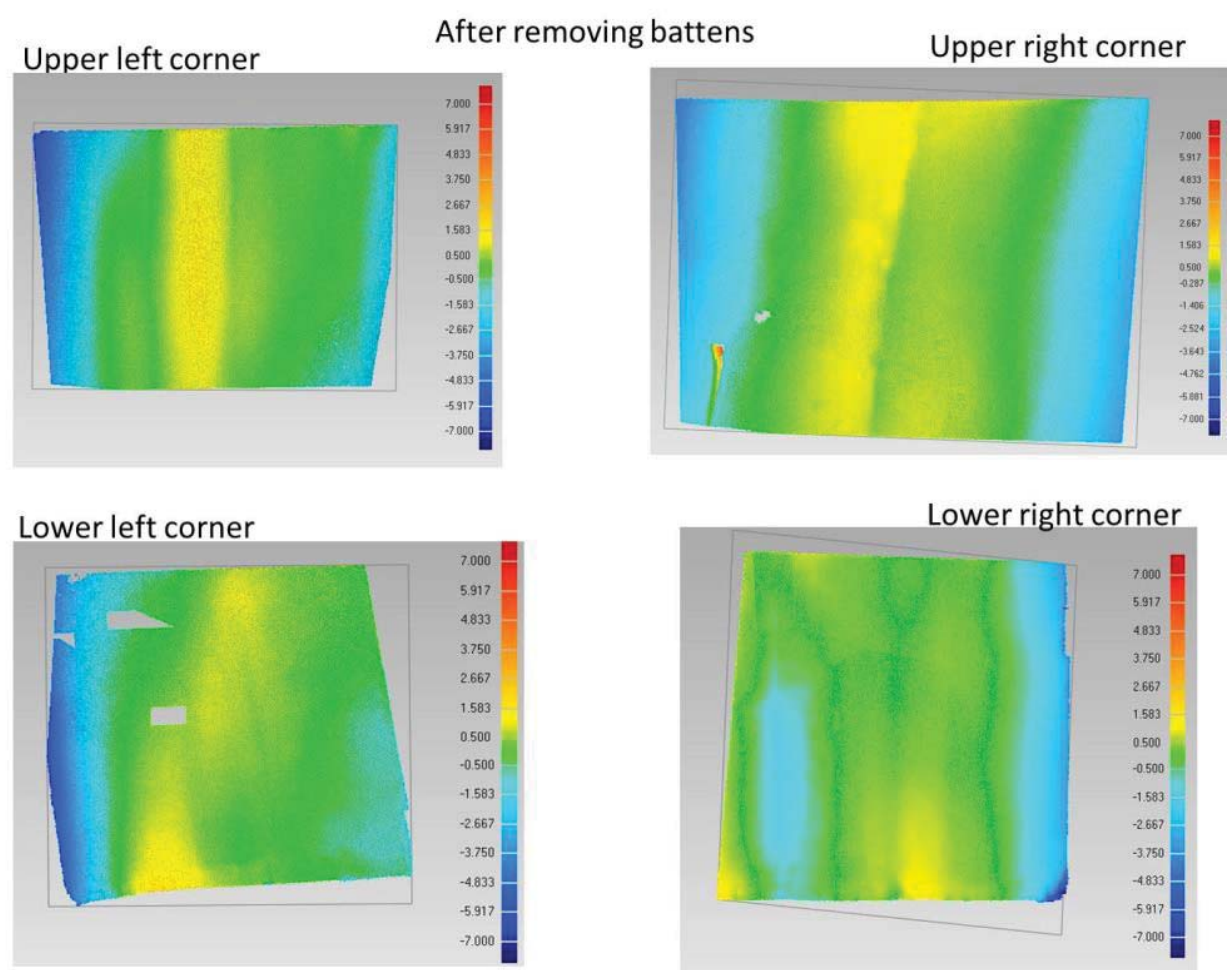

Figure 10: Image of deviations $(\mathrm{mm})$ from the best-fit plane after removing the wooden battens 


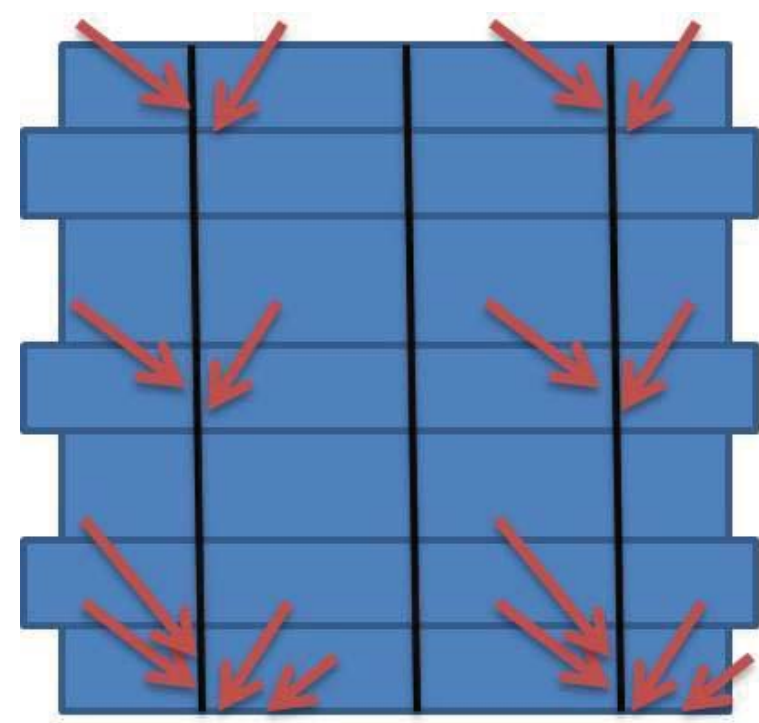

Figure 11: The Kinect sensor acquisition compared to an ideal plane shows that depth estimate varies in the image as a circular ripple

In Figure 10 is depicted the deviations from a local best-fit plane in the four corners of the painting after the battens removing, local deformation although not so pronounced $(+/-7 \mathrm{~mm})$ are to be observed, and it is clearly to be seen how the stress is focused mainly around the vertical wooden boards as expected).

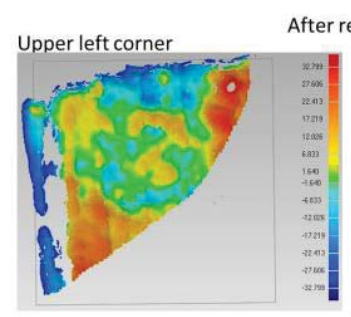

Lower left corner

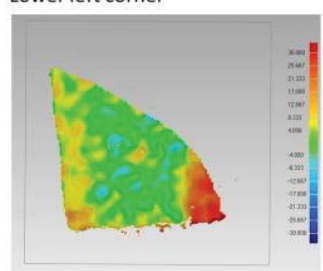

Upper right corner

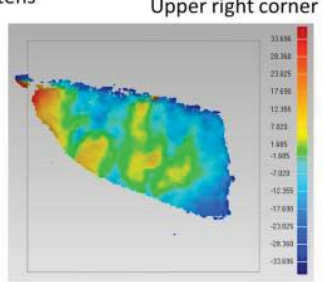

Lower right corner

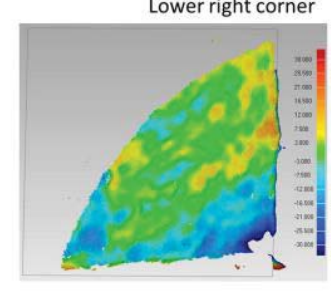

Figure 12: Kinect sensor deviations $(\mathrm{mm})$ from planarity after removing the wooden battens at the four corners

The 3D model obtained by the less expensive system, acquired after the changes due to the battens removal, still retains the basic shape of the painting and its main structures (like the vertical wooden boards). However, due to intrinsic Kinetic system distortions (like the typical circular ripple (Andersen et al. 2012) as visible in figure 8), it is not possible, to quantify the warping induced by removing the wooden battens; nonetheless this low cost depth sensor allows a qualitative evaluation of the suffered warping as shown in Figure 12 (the four corners of the 3D model after removing the battens).

\section{CONCLUSIONS}

The aim of the work presented in this paper was to assess the possibilities offered by the recently introduced Kinect system to be used as low cost monitoring device for the deformation of painting surfaces. Typically deformation of large wooden panels, which are a large part of our cultural heritage, are in the order of a few centimeters. A more professional, high end system was also employed in order to have a reference measure. The wooden panel under test was a large XV century panel which was known to be likely to undergo some deformation after the battens introduced on the back during a previous restoration treatment, were to be removed.

The test performed was carried out having in mind the simplest possibility which was a single shot acquisition form a fairly large distance. Such test showed that this low cost device can be employed for qualitative monitoring, however, as expected, no quantitative data are to be inferred. In order to have a higher accuracy more acquisitions from a shorter distance are possible, however this maybe not a suitable procedure for nonprofessionals. In order to have quantitative measures and assess the deformation properly the high end scanner proved to be much more reliable, although implying much higher costs and much longer processing time, together with the necessity of skilled and trained professionals.

\section{REFERENCES}

Andersen, M.R., Jensen, T., Lisouski, P., Mortensen, A.K., Hansen, M. K., Gregersen, T., and Ahrendt, P. (2012) "Kinect Depth Sensor Evaluation for Computer Vision Applications", Electrical and Computer Engineering Technical Report ECE-TR-6.

Guidi, G., Atzeni, C., Seracini, M., and Lazzari, S. (2004) Painting survey by 3D optical scanning. The case of Adoration of the Magi by Leonardo da Vinci. Studies in Conservation, 49(1): 1-12.

Remondino, F., Rizzi, A., Barazzetti, L., Scaioni, M., Fassi, F., Brumana, R., and Pelagotti, A. (2011), Review of Geometric and Radiometric Analyses of Paintings. The Photogrammetric Record, 26: 439-461.

Khoshelham, K., and Elberink S.O. (2012) Accuracy and Resolution of Kinect Depth Data for Indoor Mapping Applications. Sensors. 2012; 12(2):1437-1454. 
Robson, S., Bucklow, S., Woodhouse, N., and Papadaki, H. (2004) Periodic photogrammetric monitoring and surface reconstruction of a historical wood panel painting for restoration purposes. International Archives of the Photogrammetry, Remote Sensing and Spatial Information Sciences, 35(5): 395-400.
Salvi, J., Fernandez, S., Pribanic, T., and Llado, X. (2010) A state of the art in structured light patterns for surface profilometry, Pattern Recognition, Volume 43, Issue 8, August 2010, Pages 26662680.

Segal, A., Haehnel, D., and Thrun, S. (2009) Generalized-ICP. In Proceedings of Robotics: Science and Systems, Seattle, USA. 